\title{
InAsPSb quaternary alloy grown by gas source molecular beam epitaxy
}

\author{
Gene Tsai, De-Lun Wang, Chia-En Wu, Chen-Jun Wu, Yan-Ting Lin, Hao-Hsiung Lin* \\ Graduate Institute of Electronics Engineering and Department of Electrical Engineering, National Taiwan University, Taipei 10617, Taiwan
}

Available online 1 November 2006

\begin{abstract}
Quaternary $\operatorname{InAs}_{x} \mathrm{P}_{y} \mathrm{Sb}_{1-x-y}$ alloys nearly lattice-matched to InAs substrates have been successfully grown by gas source molecular beam epitaxy (GSMBE) with the composition covering the immiscibility region. Through high resolution X-ray diffractometry, we observed the compositional inhomogeneity in these alloys. Enhancement in the As incorporation in the growth can not only narrow the inhomogeneous broadening but also improve the surface morphology. Carrier recombination in band-tail states caused by the compositional inhomogeneity is attributed to the low-temperature PL emission in these samples. The PL peak energy is thus lower than the predicted band-gap energy. The energy discrepancy can be as large as $0.26 \mathrm{eV}$, and decreases dramatically to $36 \mathrm{meV}$ as the As mole fraction increases to 0.681 . For the high As mole fraction sample, band-to-band recombination is observed as the temperature is higher than $100 \mathrm{~K}$.
\end{abstract}

(C) 2006 Elsevier B.V. All rights reserved.

Keywords: A1. X-ray diffraction; A3. Molecular beam epitaxy; B1. Antimonides; B2. Semiconducting quaternary alloys

\section{Introduction}

Recently, there is an increasing demand for mid-infrared (MIR) devices in the $2-4 \mu \mathrm{m}$ wavelength range for applications such as chemical process control, toxic gases sensing, medical investigation, secure free air communication and so on [1]. Among various III-V semiconductors, quaternary InAsPSb is a promising material system for these device applications because it can be grown exactly lattice matched to InAs substrates with energy gaps covering the $2-3.5 \mu \mathrm{m}$ spectral range. A number of InAsPSb-based MIR devices such as double heterostructure lasers with InAsPSb active medium operating in the

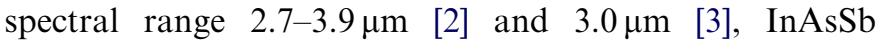
quantum well (QW) laser with InAsPSb cladding layer [4], InAs(PSb) “W” QW laser [5], InAs ${ }_{0.36} \mathrm{P}_{0.44} \mathrm{Sb}_{0.20} / \mathrm{InAs}$ heterostructure light-emitting diode for HF detection [6] and $\mathrm{InAsSb} / \mathrm{InAsPSb}$ photodetectors operating at room temperature (RT) [7] have been reported in the literature. Nonetheless, there are actually only a few reports of detailed optical characteristics from this alloy. In addition, in these previous studies, the epitaxy growth of InAsPSb

\footnotetext{
*Corresponding author. Tel.: + 886233663670 ; fax: +886223632442.

E-mail address: hhlin@ntu.edu.tw (H.-H. Lin).
}

was performed outside the extent of the miscibility gap by liquid-phase epitaxy (LPE) [2,6,7] or organometallic vaporphase deposition (OMVPE) [3-5]. The studies reported here focused on the growth of InAsPSb thick epitaxial layers matched to InAs by gas source molecular beam epitaxy (GSMBE). The composition of the quaternary covers the whole range including the extent of miscibility gap. Details of the growth conditions and characterization results are described.

\section{Experimental procedures}

A VG-V80 H GSMBE system was used to grow all the samples on (100) $n$-type InAs substrate. Pure arsine and phosphine, the precursors of the two group V gas sources, were leaked into a gas cell where the gases were cracked at $1000{ }^{\circ} \mathrm{C}$ to provide $\mathrm{As}_{2}$ and $\mathrm{P}_{2}$ beams for the epitaxy process. An Sb source was supplied by an EPI cracking effusion cell. $\mathrm{Sb}_{4}$ flux from the reservoir zone was further cracked at $1050{ }^{\circ} \mathrm{C}$ in the cracking zone to supply a mixed beam of $\mathrm{Sb}_{2}$ dimer and $\mathrm{Sb}$ monomer [8]. The group III In source came from a conventional thermal effusion cell. The fluxes of $\mathrm{Sb}$ and In sources were calibrated by an ion gauge, while the beam fluxes of As and P sources were controlled by the gas pressures of arsine and phosphine in the 
manifolds behind the micro-leaks of the gas cell. A series of $1-\mu \mathrm{m}$-thick InAsPSb quaternary layers were grown on InAs substrates at a growth temperature of $470{ }^{\circ} \mathrm{C}$ with different $\mathrm{AsH}_{3}$ pressures. The growth rate was kept at $1 \mu \mathrm{m} / \mathrm{h}$. The beam-equivalent pressure (BEP) of Sb source is fixed at half the value of In source. $\mathrm{PH}_{3}$ pressure was fixed at 990 Torr. $\mathrm{AsH}_{3}$ pressure, the only changed parameter, was adjusted from 50 to 700 Torr. The $\mathrm{PH}_{3}$ and $\mathrm{AsH}_{3}$ pressures for unity $\mathrm{V}$ to III ratio at $1 \mu \mathrm{m} / \mathrm{h}$ growth rate are 285 and 385 Torr, respectively.

The structural properties of the epilayers were characterized using a Bede QC-200 double crystal X-ray diffractometer (DXRD). Because DCXD cannot determine solely the composition of quaternary alloy, electron probe microanalysis (EPMA) was used to quantify the composition of the InAsPSb epilayers by a JEOL JXA-8200 analyzer. Photoluminescence measurements were carried out using a SPEX $500 \mathrm{M}$ monochrometer calibrated by the radiation lines of a Xe lamp and samples under test were excited by a $532 \mathrm{~nm}$ diode laser. The radiation light which passed through the monochrometer was detected by a liquid-nitrogen-cooled InSb detector. Nitrogen gas was used to purge the optical path and monochrometer to prevent atmosphere absorption (water vapor at $2.7 \mu \mathrm{m}$ and

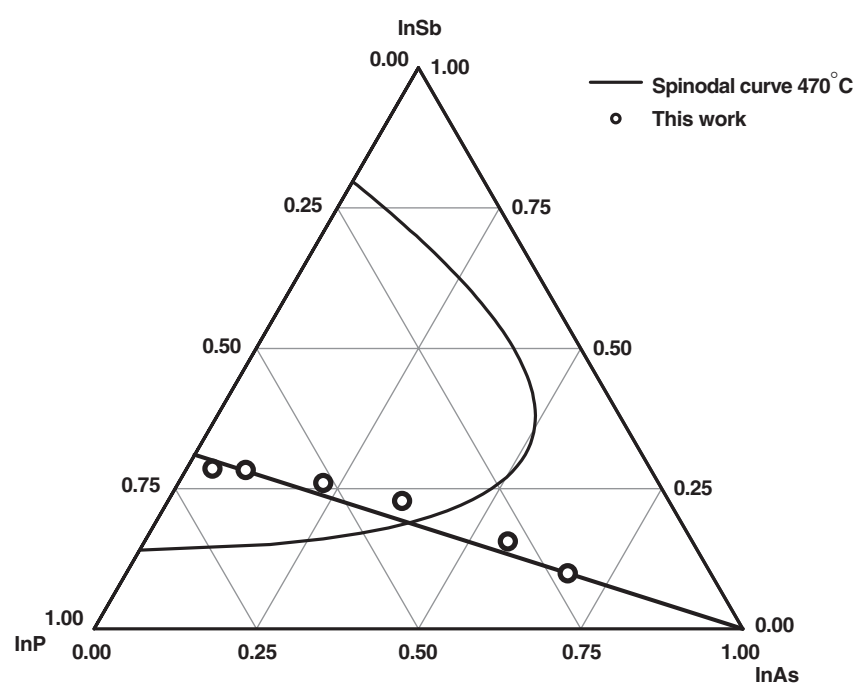

Fig. 1. Calculated spinodal isotherm for InAsPSb quaternary alloy at a growth temperature of $470{ }^{\circ} \mathrm{C}$. Solid circles represent the experimental data of this work.
$\mathrm{CO}$ at $4.1 \mu \mathrm{m})$ in the $3-5 \mu \mathrm{m}$ MIR wavelength range. $\mathrm{CaF}_{2}$ lenses and windows were also used instead of glasses for their material absorption reason.

\section{Results and discussion}

Fig. 1 shows the composition diagram for quaternary InAsPSb in which the solid circles represent the samples grown in this work. The detailed growth conditions and composition determined by EPMA are listed in Table 1 . A spinodal curve that calculated by the delta latticeparameter (DLP) model [9] for a growth temperature of $470{ }^{\circ} \mathrm{C}$ is depicted in the diagram. The spinodal curve intersects the line represents $\operatorname{InAs}_{x} \mathrm{P}_{y} \mathrm{Sb}_{1-x-y}$ lattice-match to InAs at $(x=0.39, y=0.42)$. In other words, the epilayers with As composition less than 0.39 were grown in the immiscibility region. Basically, the calculation for spinodal curve is based on the assumption of thermodynamic equilibrium. In MBE growth, which is commonly thought to be far from thermodynamic equilibrium, the growth kinetics may play an important role in determining the material structure. Furthermore, several researchers pointed out that as the depositing layer is slightly mismatched to the substrate, the effect of the resulting coherent strain may decrease the critical temperature of the spinodal point $[10,11]$. This would allow the meta-stable growth in the immiscibility region as shown in Fig. 1.

The scanning-electron-microscopy (SEM) images in Fig. 2 show the surface morphologies of the InAsPSb epilayers grown on InAs substrates. Generally, the increment in $\mathrm{AsH}_{3}$ pressure improves the surface morphology. Fig. 2(a) shows the high-density scaly defect of sample C1898 which was grown with 50 Torr $\mathrm{AsH}_{3}$ pressure. EPMA measurement indicates that the flat region in Fig. 2(a) is $\operatorname{InAs}_{0.041} \mathrm{P}_{0.664} \mathrm{Sb}_{0.295}$, which is nearly matched to the InAs substrate while the squama-like region has less $\mathrm{Sb}$ composition. As the $\mathrm{AsH}_{3}$ pressure increases to 100 and 200 Torr, eye-shape and oval-shape surface defects are observed as evidenced in Figs. 2(b) and (c). Further increase in the $\mathrm{AsH}_{3}$ pressure significantly reduces the surface defect density and gives a mirror-like surface as seen in Figs. 2(d) and (e).

The high-resolution XRD spectra are shown in Fig. 3. For $\mathrm{C} 1898$, the sample with 50 Torr $\mathrm{AsH}_{3}$ pressure, a weak broad band peaking at $\sim 400$ arcsec is observed. It reveals

Table 1

Values of quaternary growth conditions and sample compositions determined by EPMA

\begin{tabular}{lllllrr}
\hline Sample no. & $\mathrm{AsH}_{3}$ (Torr) & $\mathrm{PH}_{3}$ (Torr) & $\mathrm{In} / \mathrm{Sb}(\mathrm{BEP})$ & $T_{\mathrm{g}}\left({ }^{\circ} \mathrm{C}\right)$ & $\mathrm{As}$ & $\mathrm{P}$ \\
\hline $\mathrm{C} 1898$ & 50 & 990 & 2.03 & 470 & 0.040 & 0.675 \\
$\mathrm{C} 1899$ & 100 & & & 0.092 & 0.625 \\
$\mathrm{C} 1900$ & 200 & & & 0.223 & 0.517 \\
$\mathrm{C} 1767$ & 300 & & & 0.361 & 0.411 \\
$\mathrm{C} 1768$ & 500 & & & 0.560 & 0.283 \\
$\mathrm{C} 1770$ & 700 & & & 0.681 & 0.2284 \\
\hline
\end{tabular}



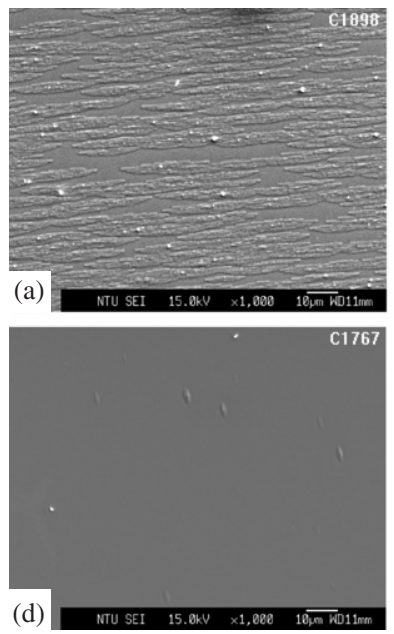
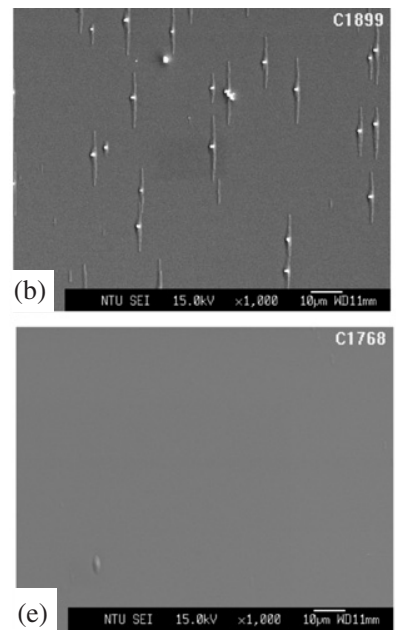
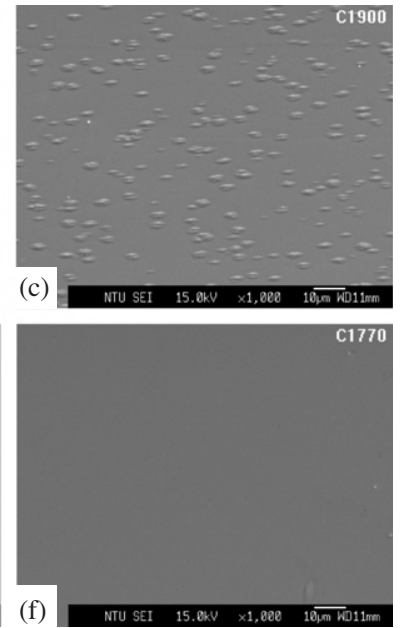

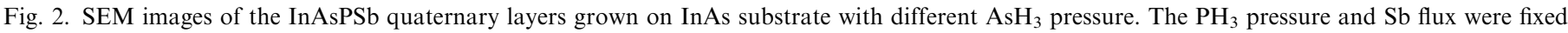


As composition clearly improves the surface morphology of the epilayers.

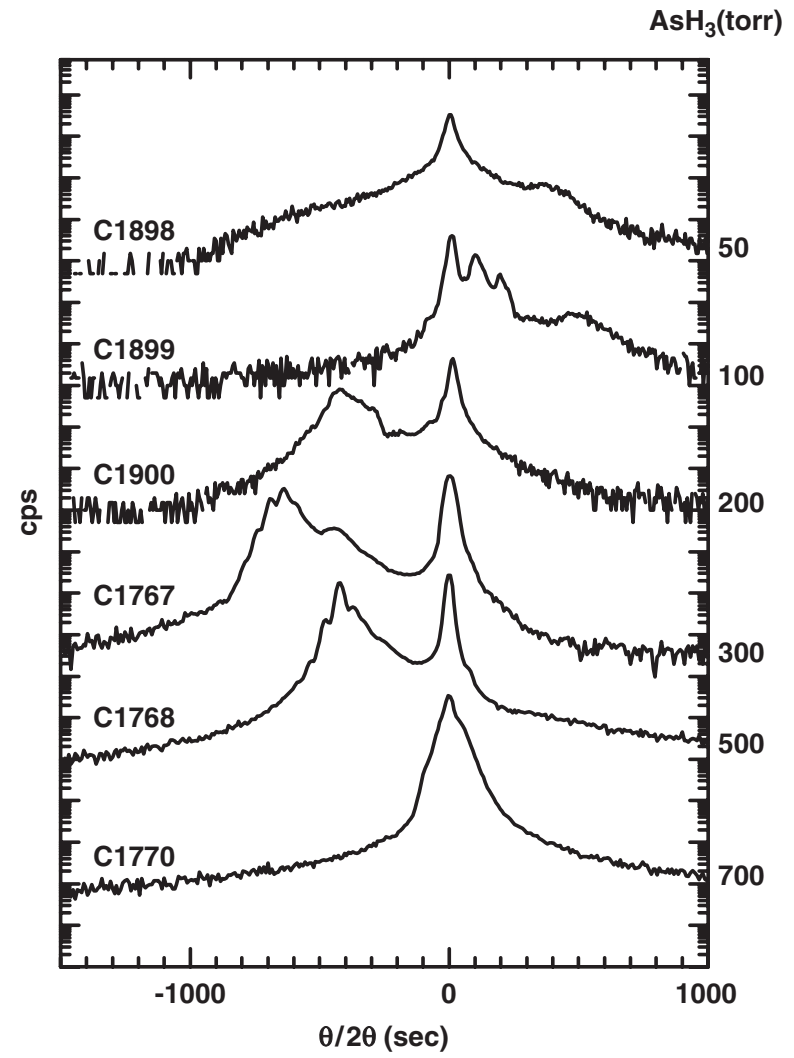

Fig. 3. X-ray diffraction spectra of the InAsPSb quaternary samples grown on InAs substrate with different $\mathrm{AsH}_{3}$ pressure.

the compositional inhomogeneity which could be due to phase separation in this sample. The increment in $\mathrm{AsH}_{3}$ pressure results in stronger and narrower peaks for the epilayers. The linewidths are less than 110 arcsec. For samples with higher As composition, the values are even $\sim 30$ arcsec, which are close to that of InAs substrate. However, there are still tiny peaks embedded in the shoulders of the main peak in these samples. Notice that the intensity of the figure is in logarithmic scale. It suggests that there are still compositional inhomogeneities in the samples with $\mathrm{AsH}_{3}$ pressure higher than 50 Torr. However, the higher the As composition in the samples, the softer the embedded peaks. For C1770, the sample with the highest As composition, extra tiny peaks are hardly recognized. In other words, the incorporation of As helps to improve the homogeneity in the epilayers.

Photoluminescence measurements were also performed on these quaternary samples to investigate their optical properties. The low-temperature PL $(T=10 \mathrm{~K})$ peak energy of the quaternary samples and the calculated InAs-matched quaternary band-gap energy are plotted against the As concentration in Fig. 4. The calculations are based on the band-gap energies of the related binaries and the bowing parameters between them [12-14]. As can be seen in the figure, all the PL peak energies are lower than the calculated band-gap energy. In addition, as the As composition increases, instead of monotonically decreasing predicted by the band-gap energy calculation, the trend of the PL peak energy is irregular. The energy difference between the PL energy and the calculated band-gap energy has the highest value, $0.26 \mathrm{eV}$, for sample $\mathrm{C} 1898$ with the $\mathrm{AsH}_{3}$ pressure of 50 Torr. When the As composition in the epilayer increases, the energy separation decreases. For samples with the composition outside the miscibility gap, the difference in energy reduces significantly to $\sim 36 \mathrm{meV}$. A similar phenomenon has been observed in InPSb samples grown by OMVPE previously [13]. The reported energy difference was as high as $0.24 \mathrm{eV}$ and was attributed to the carrier recombination in the tail states resulting from compositional clusters. In this study, the aforementioned compositional inhomogeneity observed from XRD spectra and surface morphology is in favor of this attribution. The potential fluctuation in the epilayers due to the compositional inhomogeneity results in localized band tail states. The more serious the inhomogeneity, the broader the band 


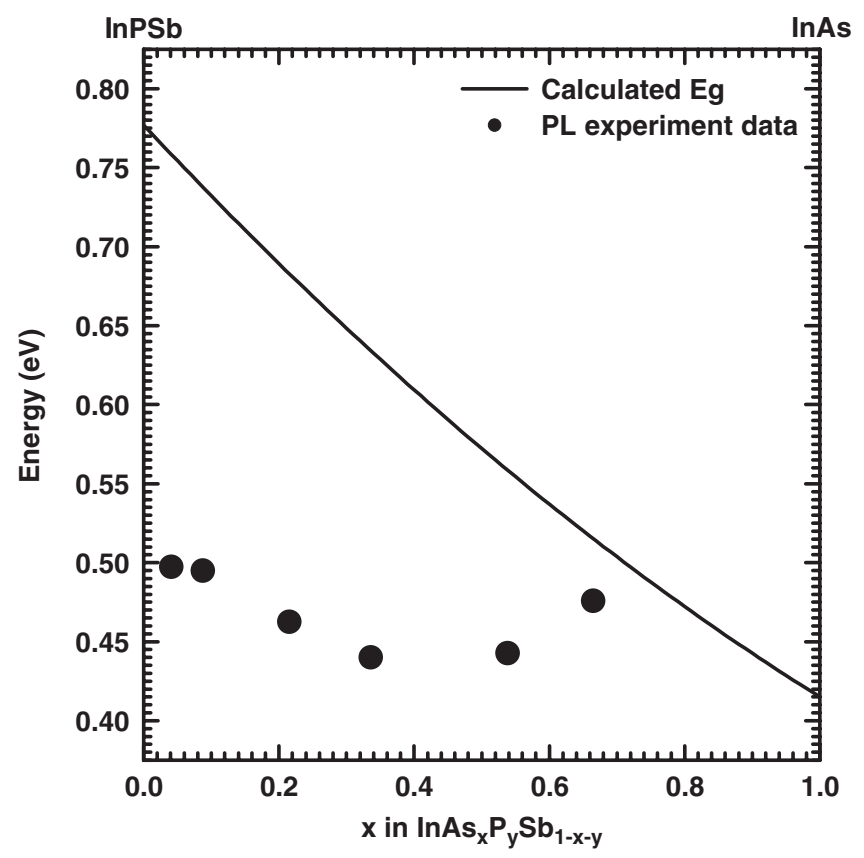

Fig. 4. $10 \mathrm{~K}$ PL transition energy and the calculated band-gap energy of the InAsPSb epilayers as functions of As composition.

tail states, and the larger the energy separation. Furthermore, previous studies in the literature [15] suggested that the band alignment between InAsPSb and InAs is type II. Therefore, it is reasonable to assume the same type of band alignment between InAsPSb alloys with different composition. This type-II band line-up would cause the spatial separation of electrons and holes and result in space-charge fields between different clusters. The extra electric field could broaden the band-tail states. Furthermore, the recombination of the spatially separated electron and hole at the cluster interface has the transition energy much lower than the corresponding InAsPSb band-gap energies. Band-tail states due to intrinsic alloy fluctuation and typeII band line-up may explain the extraordinarily large energy separations in this study and Ref. [13].

Fig. 5 shows the temperature-dependent PL spectra for $\mathrm{C} 1770$, the sample with the narrowest XRD line width and the smallest energy difference. The dips in the spectra near $2700 \mathrm{~nm}$ are associated with water vapor absorption in the optical path of the detection system. We attribute the peak at low temperature to the recombination in the band-tail states resulting from alloy fluctuation. This peak quenches quickly as temperature rises. Another higher energy peak emerges and dominates the PL recombination process as the temperature reaches $97 \mathrm{~K}$. The peak shows, red shift as the temperature further increases to $247 \mathrm{~K}$. Fig. 6 shows the PL peak energy of $\mathrm{C} 1770$ as a function of temperature. A calculated band-gap energy curve is also plotted in the figure. The temperature dependency of the curve is based on the famous Varshni equation [16] with the $\alpha$ and $\beta$ constants interpolated linearly from those of the binaries consisting of the alloy [12]. As can be seen in the figure, the experimental peaks are in reasonably good agreement with

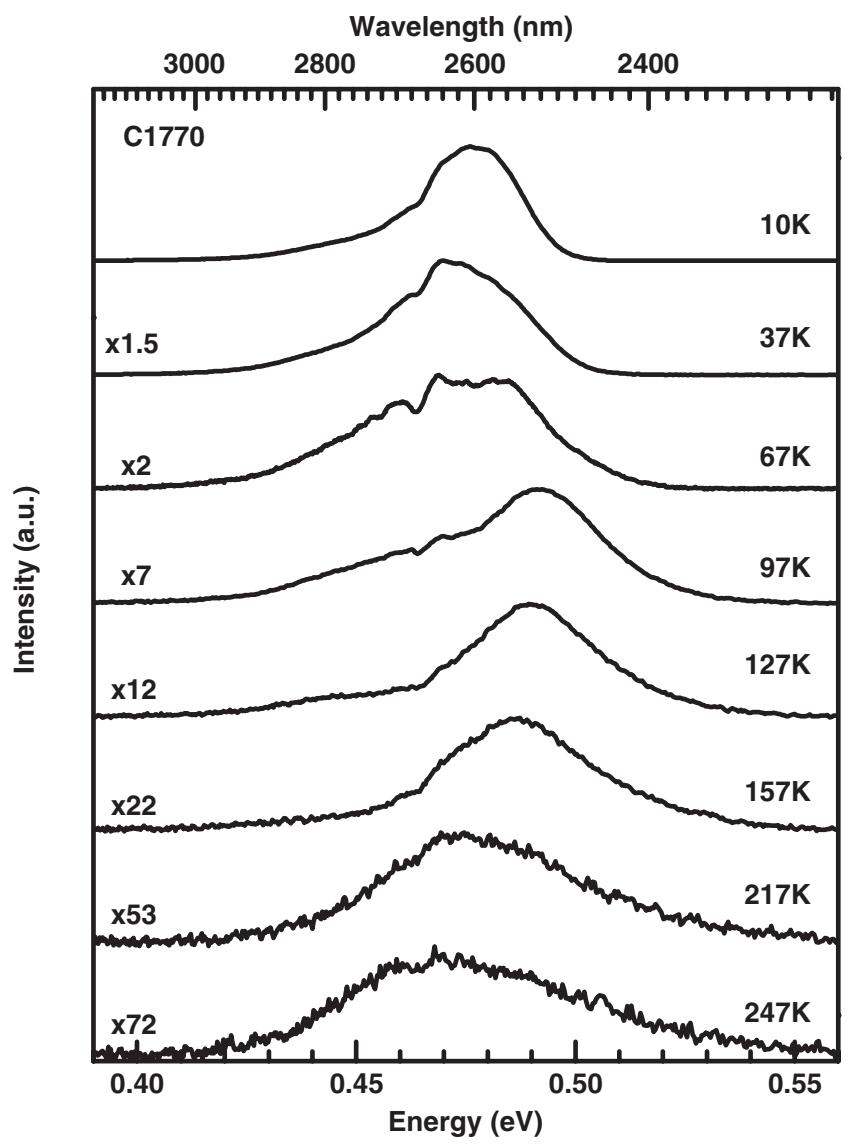

Fig. 5. PL spectra of sample $\mathrm{C} 1770\left(\operatorname{InAs}_{0.681} \mathrm{P}_{0.220} \mathrm{Sb}_{0.099}\right)$ at $10,37,67$, 97, 127, 157, 217 and $247 \mathrm{~K}$. The PL spectra are normalized for clarity. The dip of the spectrum is due to water vapor absorption at $2.7 \mu \mathrm{m}$.

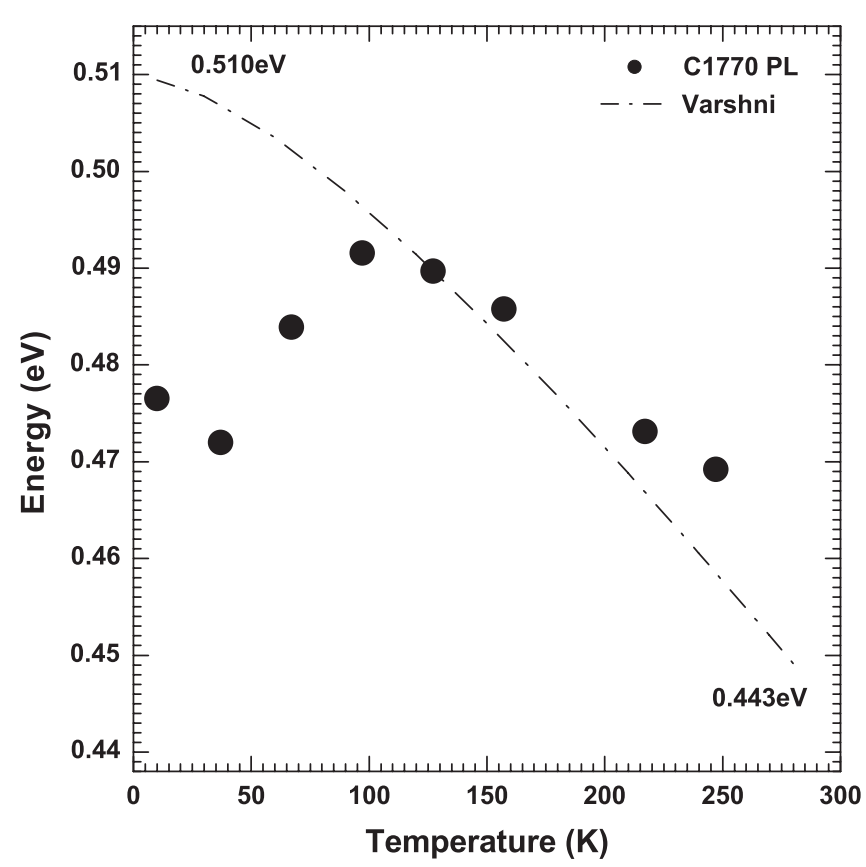

Fig. 6. A comparison of the temperature dependence of the PL transition energy and calculated quaternary band-gap using the Varshni equation. 
the calculated curve when the temperature is higher than $97 \mathrm{~K}$. Therefore, we assign the high-energy peak to the band-to-band recombination in the alloy. When $T=10 \mathrm{~K}$, the PL is dominated by the recombination in the tail states because the $36 \mathrm{meV}$ energy difference is a deep potential barrier at this temperature for the localized carriers or excitons. Since the density of state in the band tail is much lower than those in the conduction and valence bands, the carriers are rapidly thermalized to the bands, resulting in band-to-band emission, as the temperature rises to $97 \mathrm{~K}$. We also performed a power-dependent PL study on this sample at $10 \mathrm{~K}$. As the excitation power increases from $0.37 \mathrm{~mW}$ to $112 \mathrm{~mW}$, a blue shift of $18 \mathrm{meV}$ in PL peak energy was observed. This large blue shift is due to the rapid filling of the localized band-tail states and is another evidence for band-tail state recombination.

\section{Conclusion}

In summary, quaternary InAsPSb epilayers nearly lattice-matched to InAs substrate were successfully grown by GSMBE. Compositional inhomogeneity in the epilayers was observed through high-resolution X-ray diffractometry. The increment in the As mole fraction of the alloy may improve both the surface morphology and inhomogeneous broadening caused by alloy fluctuation. Low-temperature PL study reveals a difference between PL peak energy and calculated band-gap energy, which is due to the PL emission from the band-tail states resulting from compositional fluctuation. The discrepancy in energy decreases as the As mole fraction increases. For a sample with high As mole fraction, band-to-band recombination is observed as the temperature is higher than $100 \mathrm{~K}$.

\section{Acknowledgments}

The authors would like to thank Prof. A. Krier for his valuable discussion. G. Tsai wishes to acknowledge the assistance of Frank Tseng, Visual Photonics Epitaxy Co., Ltd with the DXRD measurement. This work was supported by the National Science Council of Republic of China under contract no. NSC 94-2215-E-002-016.

\section{References}

[1] J. Wagner, C.H. Mann, M. Rattunde, G. Weimann, Appl. Phys. A 78 (2004) 505 .

[2] A.N. Baranov, A.N. Imenkov, V.V. Sherstnev, Yu.P. Yakovlev, Appl. Phys. Lett. 64 (1994) 2480.

[3] N. Kobayshi, Y. Horikoshi, Jpn. J. Applied Physics 19 (1980) L641.

[4] B. Lane, D. Wu, A. Rybaltowski, H. Yi, J. Diaz, M. Razeghi, Appl. Phys. Lett. 70 (1997) 443.

[5] A. Joullie, et al., Appl. Phys. Lett. 76 (2000) 2499.

[6] A. Krier, Y. Mao, IEE Proc.-Optoelectron. 144 (1997) 355.

[7] X.Y. Gong, H. Kan, T. Makino, T. Iida, Y. Gao, M. Aoyama, M. Kumagawa, T. Yamaguchi, Jpn. J. Appl. Phys. 38 (1999) 685.

[8] Y. Rouillard, B. Lambert, Y. Toudic, M. Baudet, M. Gauneau, J. Crystal Growth 156 (1995) 30.

[9] G.B. Stringfellow, J. Crystal Growth 27 (1974) 21.

[10] M. Quillec, C. Daguet, J.L. Benchimol, H. Launois, Appl. Phys. Lett. 40 (1982) 325

[11] D. Schenker, T. Miyamoto, Z. Chen, M. Kawaguchi, T. Kondo, E. Gouardes, J. Gemmer, C. Emmer, F. Koyama, K. Iga, Jpn. J. Appl. Phys. 39 (2000) 5751.

[12] V. Swaminathan, A.T. Macrander, Materials aspects of GaAs and InP based structures, Prentice Hall, New Jersey, 1991, p. 10.

[13] E.H. Reihlen, M.J. Jou, Z.M. Fang, G.B. Stringfellow, J. Appl. Phys. 68 (1990) 4604.

[14] M. Krijn, Semicond. Sci. Technol. (1991) 27.

[15] A. Krier, Phil. Trans. R. Soc. Lond. A 359 (2001) 599.

[16] Y.P. Varshni, Physica 34 (1967) 149. 